\title{
Effect of COVID-19 on HIV, tuberculosis, and prevention of mother-to-child transmission of HIV indicators in Mopani district, South Africa
}

\author{
C Mutyambizi, ${ }^{1} \mathrm{PhD}$; J Dunlop, ${ }^{1,2} \mathrm{MB}$ BCh, MSc (Med) Child Health; C Maluleke, ${ }^{2} \mathrm{MB}$ ChB; L Ranoto, ${ }^{1} \mathrm{MB}$ ChB; \\ T Chetty, ${ }^{3}$ MB ChB, FCPHM (SA), MMed (PHM), PhD; R Ndou, ${ }^{1}$ EN; H Struthers, ${ }^{1,4} \mathrm{PhD}$; J A Mcintyre, ${ }^{1,5}$ MB ChB, FRCOG; \\ K Rees, ${ }^{1,6} \mathrm{MB}$ BCh, FCPHM (SA) \\ ${ }^{1}$ Anova Health Institute, Johannesburg, South Africa \\ ${ }^{2}$ Division of Community Paediatrics, School of Paediatrics, Faculty of Health Sciences, University of the Witwatersrand, Johannesburg, South Africa \\ ${ }^{3}$ HIV Prevention Research Unit, South African Medical Research Council, Durban, South Africa \\ ${ }^{4}$ Division of Infectious Diseases and HIV Medicine, Department of Medicine, Faculty of Health Sciences, University of Cape Town, South Africa \\ ${ }^{5}$ School of Public Health and Family Medicine, Faculty of Health Sciences, University of Cape Town, South Africa \\ ${ }^{6}$ Department of Community Health, School of Public Health, University of the Witwatersrand, Johannesburg, South Africa
}

Corresponding author: C Mutyambizi (mutyambizic@anovahealth.co.za)

\begin{abstract}
Background. To prevent the spread of SARS-CoV-2, many countries instituted lockdown measures. As the virus was initially slow to spread to rural areas in South Africa, Mopani district in Limpopo Province did not experience a high incidence of COVID-19 until the second wave in December 2020. Until then, lockdown measures were more likely than SARS-CoV-2 infections to have an adverse impact on health services. Objectives. To analyse HIV, tuberculosis (TB) and prevention of mother-to-child transmission of HIV (PMTCT) indicator trends in Mopani during the COVID-19 lockdown and two COVID-19 waves.

Methods. Using monthly data from the District Health Information System from February 2019 to December 2020, we conducted a retrospective review of data elements and indicators that fall into the following domains: primary healthcare head count (HC), HIV, antiretroviral treatment (ART), PMTCT and TB. Aggregated data were analysed, and an interrupted time series analysis was conducted. We assessed percentage changes between the January - March 2020 and April - June 2020 periods, and differences in means for the period April - December 2019 v. the period April - December 2020 were assessed for statistical significance.

Results. At the start of the first wave in April 2020, a statistically significant decline of $10 \%$ was recorded for total HC utilisation rates $(p=0.1$ ). We also found declines of 665 HIV tests (from 1608 to 942) and 22 positive HIV tests (from 27 to 4 ) for children between the ages of 18 months and 14 years $(p=0.05)$, with no recovery. Significant declines were also recorded for children aged $<15$ years starting (change from 35 to 21) and remaining (change from 4032 to 3 986) on ART, as well as for adults starting ART (change from 855 to 610 ) at the onset of the first wave $(p=0.01)$. No significant change was detected in PMTCT and TB indicators during the first wave. Pronounced decreases in HC were recorded in December, during the country's second wave (change from 237965 to 227 834).

Conclusion. Declines were recorded for most indicators in Mopani, with HC being affected the most at the start of the first wave and not showing any significant recovery between waves. Strategies are required to mitigate the effects of future COVID-19 waves and encourage positive health-seeking behaviour.
\end{abstract}

S Afr Med J 2021;111(12):1181-1189. https://doi.org/10.7196/SAMJ.2021.v111i12.15822

The first confirmed case of COVID-19 in South Africa (SA) was reported on 5 March 2020. ${ }^{[1,2]}$ Since then, SA has become the most affected country in Africa, with 2.9 million cases and $>89000$ deaths due to COVID-19 as at 1 November 2021. ${ }^{[3]}$ SA experienced a high number of COVID-19 infections in the first wave, peaking in July. The daily cases then declined before a major surge in December, when the country experienced its second wave of increased cases, probably fuelled by a new variant of the virus. ${ }^{[4]}$ Like many other countries globally, SA implemented an unprecedented national shutdown to combat the spread of the virus. ${ }^{[2,5]}$ The implementation of the National State of Disaster on 15 March gave the government the power to carry out and implement what later became a five-level COVID-19 alert system. Of the five levels of restrictions, the highest, most restrictive is alert level 5 and the lowest alert level 1. Level 5 lockdown measures included drastic restrictions on movement and the closure of all non-essential activities. During level 1, most normal activities were allowed to take place with precautions and adhering to health guidelines. ${ }^{[6]}$ At the onset, on 27 March, SA went into alert level 5 and over the months that followed gradually eased to level 1 in September (at the end of the first wave) and then back to level 3 in December (during the second wave). ${ }^{[1,7]}$

SA is described as having a quadruple burden of disease resulting from non-communicable diseases (such as diabetes and hypertension), communicable diseases (such as HIV/AIDS and TB), an epidemic of maternal, newborn and child illnesses, and violence and injury. ${ }^{[8]}$ The emergence of COVID-19 has placed additional pressure on an already strained healthcare system and has resulted in changes both in demand for and supply of healthcare generally. ${ }^{[9,10]}$ On the demand side, public anxiety and fear of contracting COVID-19 have resulted in patients postponing care. Lockdown restrictions disrupted public transport services that clients use to access health facilities, and the indirect effects of the economic downturn have made healthseeking less manageable. With regard to supply, there has been a shift of resources from other healthcare issues, as hospital wards 
and human resources were allocated to COVID-19 care. Decisions were taken to increase the intervals between client appointments, allowing chronic clients to receive 2 months (or even $>2$ months) of medication at a time. In some cases, clinics were temporarily closed for disinfection following a positive COVID-19 case in a staff member. ${ }^{[9,10]}$ In Limpopo Province, all antiretroviral treatment (ART) clients previously managed at hospitals were referred to primary healthcare clinics to increase space for clients with COVID-19.

Accounts of the impact of the COVID-19 pandemic on healthcare in SA are sparse and varying in available literature. ${ }^{[2,9,11-14]}$ Among studies that assessed HIV-related indicators, Siedner et al..$^{[13]}$ found no change in HIV-related clinic visits at the start of the lockdown, while Burger et al. ${ }^{[9]}$ and Pillay et al. ${ }^{[2]}$ found reductions in HIV testing and Dorward et al. ${ }^{[12]}$ found that, while ART provision was sustained, HIV testing and ART initiations were heavily affected by the lockdown. The present study adds to this literature by focusing on the HIV, tuberculosis (TB) and prevention of mother-to-child transmission of HIV (PMTCT) indicator trends in Mopani district in Limpopo across two response points: ( $i$ ) at the beginning of the first lockdown (level 5) in April 2020; and (ii) at the move to lockdown level 1 in September 2020.

A study of this nature is important for various reasons. HIV and TB remain among the top 10 leading causes of death in SA, accounting for $11.2 \%$ of deaths in 2017. ${ }^{[15]} \mathrm{SA}$ is reported to have one of the largest HIV/ART programmes in the world. ${ }^{[16]}$ Of concern, reduced health-seeking behaviour by patients due to COVID-19 could reverse the gains from other healthcare priorities, as they are neglected in favour of addressing COVID-19. ${ }^{[10]}$ Modelling studies have shown that an interruption to healthcare access could result in additional HIV-related deaths. ${ }^{[17]}$ Secondly, there is evidence that lockdown interventions may have had varying effects in SA provinces, with health services not being similarly affected. ${ }^{[2]}$ There is a paucity of research on how COVID-19 lockdown measures affected HIV, TB and PMTCT health-seeking behaviour, particularly in outlying areas. Early in the pandemic, COVID-19 was slow to spread to smaller and rural areas. As such, Mopani did not experience a high incidence of COVID-19 until the second wave in December 2020. Until then, lockdown measures were more likely than SARS-CoV-2 infections to have an effect on health services. A study of this nature is important for facilitating actions and strategies to be put in place to mitigate the effects of future COVID-19 waves and encourage health-seeking behaviour.

\section{Methods}

\section{Study area}

The study was conducted in Mopani District Municipality. Mopani district is one of the five districts in Limpopo Province, SA. According to Mopani's Integrated Development Plan, the district has a population of $\sim 1.1$ million. ${ }^{[18]}$ One of the most rural districts in SA, the region is sparsely populated, with the majority pf people living in rural areas (81\%) ${ }^{[18]}$ Approximately $55 \%$ of the population are female, $49 \%$ are aged $0-19$ years, and $39 \%$ are unemployed. ${ }^{[18]}$ Healthcare in the public sector is provided through a mix of primary healthcare clinics, mobile clinics and hospitals. ${ }^{[18}$

During the first COVID-19 wave in SA, cases peaked at $~ 292$ per 100000 population in July 2020 before gradually decreasing to a nadir of 64 per 100000 in November and then spiking to 390 per 100000 as the country experienced its second wave. ${ }^{[19]}$ At the pandemic onset, large urban areas were most affected, with slow spread to smaller towns and rural areas, such as Mopani district. As such, Mopani did not experience a high incidence of COVID-
19 during the first wave in April. As the months progressed, new COVID-19 cases in the district increased from 13.3 per 100000 population in June to 69.2 per 100000 population in August. At the end of the first wave, as the country eased into lockdown level 1 in September, cases dropped to 33.5 per 100000 population. At the time of the beginning of the second wave in December, new cases stood at 58.9 per 100000 population.

The weekly report on deaths in SA published by the South African Medical Research Council reported $>157500$ excess deaths in SA and >14 500 excess deaths in Limpopo over the period 3 May 2020 1 May 2021. ${ }^{[20]}$ Excess deaths are defined as deaths that occur above normal expectations. ${ }^{[20]}$ The reported COVID deaths and excess deaths for Limpopo showed a drastic increase as the country went through the second wave. It is estimated that a substantial proportion of these deaths were linked to COVID-19.

\section{Data}

This study made use of monthly aggregated data from the District Health Information System (DHIS) from February 2019 to December 2020. Outliers were identified and numbers verified and corrected using the January 2021 DHIS export. The DHIS dataset for Mopani includes data from all health facilities in the district. Ethical approval was obtained from the Human Sciences Research Council Research Ethics Committee (ref. no. 3/22/08/18) and the Limpopo Provincial Research Committee. Data were then accessed from the DHIS records.

\section{Study design}

For this study, we conducted a descriptive analysis of the outcome variables. We assessed percentage changes between the January March 2020 and April - June 2020 periods, and differences in means for the period April - December 2019 v. the period April - December 2020 were assessed for statistical significance.

We then performed an interrupted time series analysis (ITSA) to estimate the changes in various indicators in Mopani district across two response points.

\section{Statistical analyses}

We conducted a single-group ITSA regression model as described by Linden, ${ }^{[21]}$ which takes on the following regression model:

$$
Y_{t}=\beta_{0}+\beta_{1} T_{1}+\beta_{2} X_{t}+\beta_{3} X_{t} T_{t}+\varepsilon_{t}
$$

$T_{t}$ is the time that has lapsed since the start of the study. In our study, the outcome variables are taken monthly. $Y_{t}$ represents the outcome variable measured at each time point $t ; X_{t}$ is the dummy variable which takes on the value of 1 in the post-intervention period and a value of 0 in the pre-intervention period (the intervention in our study is the lockdown); and $X_{t} T_{t}$ is the interaction between time and intervention.

$\beta_{0}$ represents the starting levels for the outcome variable at $T=0$; $\beta_{1}$ is the trend in the outcome variable during the pre-intervention period; $\beta_{2}$ is the change in the level of the outcome variables following the introduction of the intervention; and $\beta_{3}$ represents the change in the slope after the intervention. If the slope in the trend line before the lockdown is the same as the slope after the lockdown, it would mean that the trend for the outcome variable remained the same despite the lockdown. An increase in the slope after the lockdown indicates an upward trend in the data, whereas a decrease in the slope suggests a downward trend. In our study, we hypothesised both a level and a slope change, so we applied the full model as presented in equation 1. Significant $p$-values in $\beta_{2}$ and $\beta_{3}$ indicate an 
immediate lockdown effect and a lockdown effect over time. In the model estimation, we assumed the presence of serial autocorrelation of order 1 and then conducted a post estimation test using 'actest' by Baum and Schaffer ${ }^{[22]}$ and adjusted the models to account for any autocorrelation.

We used the Stata statistical software package, version 14.2 (StataCorp, USA). Interrupted monthly time series plots show outcome variable data as dots and data regression (straight line).

\section{Outcome variables}

Our primary outcomes of interest were: (i) primary healthcare head count (HC) indicators; (ii) HIV services indicators; (iii) ART indicators; (iv) PMTCT indicators; and ( $v$ ) TB treatment indicators. $\mathrm{HC}$ indicators included in the analysis a total for all age groups, as well as disaggregated by age: $<5$ years, 5 - 9 years, $10-19$ years and $\geq 20$ years. For the ITSA, all HC indicators were converted to utilisation rates using the population estimates for Mopani, as provided in Statistics South Africa's 2016 Community Survey and the district's Integrated Development Plan. ${ }^{[18,23]}$

Elements included under the HIV domain were also disaggregated by age, including polymerase chain reaction (PCR) tests and PCR tests positive (representing testing for babies aged $<18$ months); HIV tests and HIV tests positive for 18 months - 14 years; and HIV tests and HIV tests positive for $\geq 15$ years. ART indicators included were also disaggregated by age into children $(<15$ years) and adults ( $\geq 15$ years) starting and remaining on ART. PMTCT indicators of interest included antenatal visits, total pregnant HIV-positive women identified, and number of HIV-positive pregnant women started on ART. TB elements included TB tests positive, TB treatment total, TB treatment $<5$ years and TB treatment $\geq 5$ years.

\section{Results}

Changes in data elements included in the analysis are shown in Table 1. While descriptive analysis of the data is useful and shows changes across indicators as a result of the lockdown, the ITSA can isolate the effect of the lockdown periods on routine service delivery. Results from the ITSA are shown in Table 2, which shows that ITSA results are not homogeneous for the health indicators. The ITSA for routine service delivery at the beginning of the April lockdown and at the end of the first wave in September for some selected indicators are also graphically represented in Fig, 1 . The dashed vertical lines in the figure represent the start of the lockdown in April 2020 and the end of the first wave or start of the level 1 lockdown in September.

\section{Primary healthcare head count indicators}

$\mathrm{HC}$ is an indicator of health facility visits regardless of the reason for health-seeking, or the number of times an individual seeks care. Data show that the mean HC for the period April - December 2019 was significantly higher than that for April - December 2020 for all HC indicators (Table 1) $(p<0.05)$. Quarterly data for April - June $2020 \mathrm{v}$. January - March 2020 also showed declines across all HC indicators, with the 5 - 9 years age group experiencing the largest drop (39\%). Drops in visits were identified immediately following the start of lockdown in April 2020. The HC indicator did not appear to improve, even with the change to level 1 in September 2020. Further decreases were seen for HC indicators in December 2020, during the country's second wave.

The ITSA shows that the April 2020 lockdown was associated with a statistically significant decline in all $\mathrm{HC}$ indicators except the $\geq 20$ years category. To illustrate this decline, we have included graphs for the HC total and HC 5 - 9 years age group (see Fig. 1).
The immediate decline in HC utilisation rates for the 5 - 9 years age group is then followed by a statistically significant decrease in the monthly trend $(p=0.01)$. We then see that at the end of the first wave, as the country eased into lockdown level 1 there was a statistically significant increase in HC utilisation rates for the 5 - 9 years age group. We identified a non-significant increase immediately following the move to lockdown level 1 for the total HC utilisation rates indicator, the $10-19$ years age group and the $>20$ years age group. We also identified a non-significant decrease for the $<5$ years age group. Level 1 was associated with statistically significant decreases in the monthly trend for total HC utilisation rate, including those for $10-19$ years and $\geq 20$ years (Fig. 1). Level 1 was also associated with a statistically significant increase in the 5 - 9 years age group. There were no significant changes in trend over time for the HC utilisation groups and the $<5$ years group following the move to level 1 .

\section{HIV indicators}

Data show that the HIV tests and HIV tests positive elements for children aged $>18$ months dropped significantly for the April December 2020 period when compared with April - December 2019 (Table 1) $(p=0.05)$. The largest reduction occurred for HIV tests positive in the age group 18 months - 14 years, which dropped from a monthly mean of 27 to 11 . While all other HIV indicators were affected by the April lockdown, PCR tests and PCR tests positive appeared to be unaffected. Following the huge dip in April 2020, adult positives showed an upward trend before decreasing again after September 2020. Except for the positive PCR tests, quarterly data for April - June 2020 v. January - March 2020 also showed declines across all HIV indicators.

Results in Table 2 indicate that HIV tests and HIV tests positive for children aged $>18$ months were negatively affected by the April lockdown (also see Fig. 1). During the first month of the lockdown, there appeared to be significant decreases in HIV tests for the age group 18 months -14 years $(n=665 ; p=0.05)$, HIV tests positive for the age group 18 months - 14 years $(n=22 ; p=0.01)$, HIV tests for the age group $\geq 15$ years ( $n=10595 ; p=0.01$ ), and HIV tests positive for the age group $\geq 15$ years $(n=277 ; p=0.01)$. These decreases were then followed by statistically significant increases in the monthly trend. PCR tests and PCR tests positive were unaffected in the month of the lockdown, but showed statistically significant decreases in the monthly trend after the lockdown. The change to lockdown level 1 in September resulted in statistically significant decreases in HIV tests in the age group 18 months -14 years $(n=597 ; p=0.01)$, HIV tests in the age group $\geq 15$ years $(n=7056 ; p=0.01)$ and HIV tests positive in the age group $\geq 15$ years $(n=195 ; p=0.01)$, which were followed by statistically significant decreases in the monthly trends of 213 for HIV tests in the age group 18 months - 14 years $(p=0.01), 3520$ for HIV tests in the age group $\geq 15$ years $(p=0.01)$ and 31 for HIV tests positive in the age group $\geq 15$ years $(p=0.1)$. To illustrate this change, we have included graphs for HIV tests in the age group 18 months 14 years and HIV tests in the age group $\geq 15$ years in Fig. 1 .

\section{ART indicators}

Data show statistically significant declines in adults and children starting ART over the April - December 2020 period v. the April December 2019 period $(p=0.01)$. Quarterly data for April - June 2020 v. January - March 2020 also showed declines across these indicators, with children aged $<15$ starting ART experiencing the largest drop (45\%). As shown in Table 1, adults naive starting ART (33\%), children naive starting ART (41\%) and children remaining on 


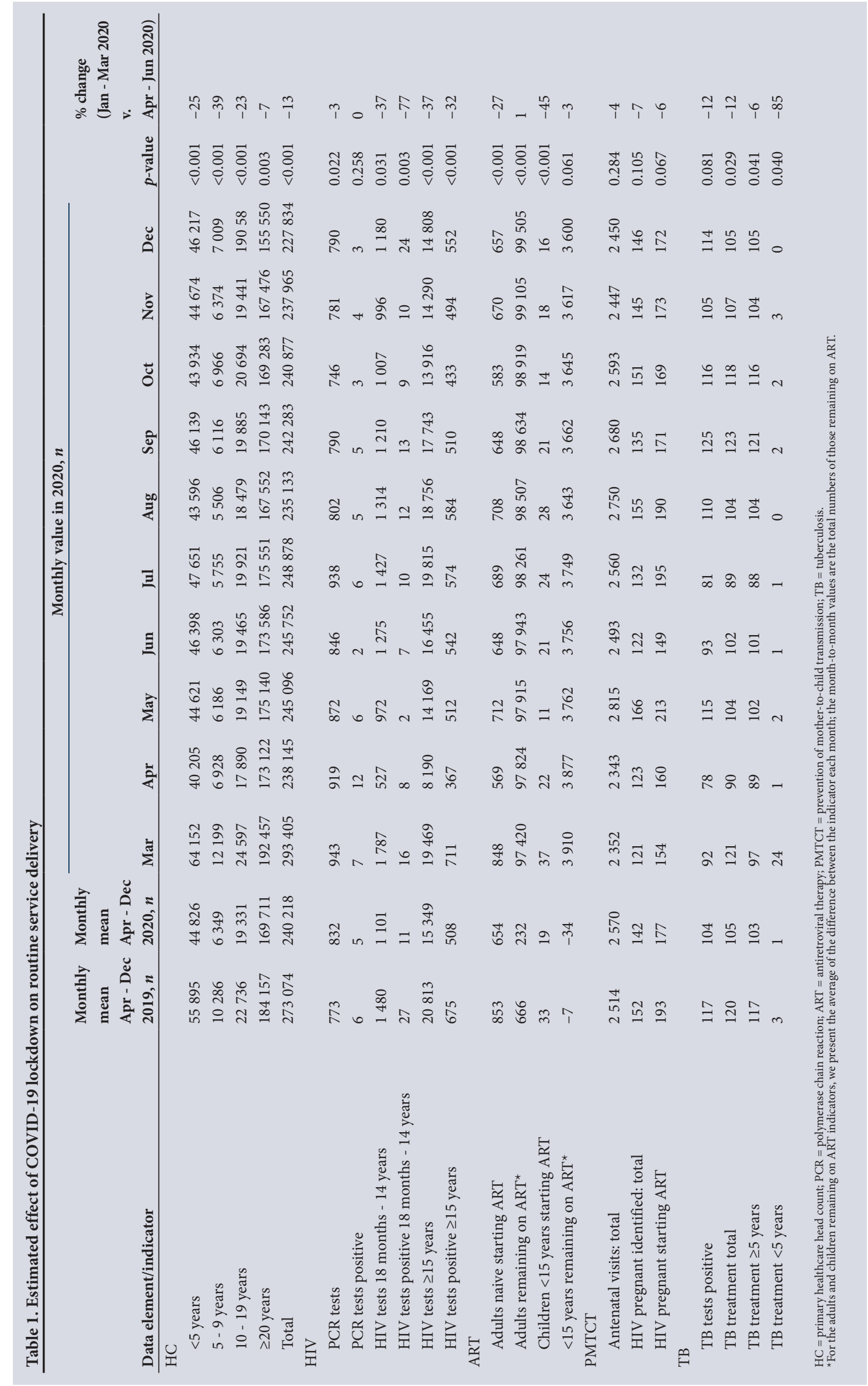




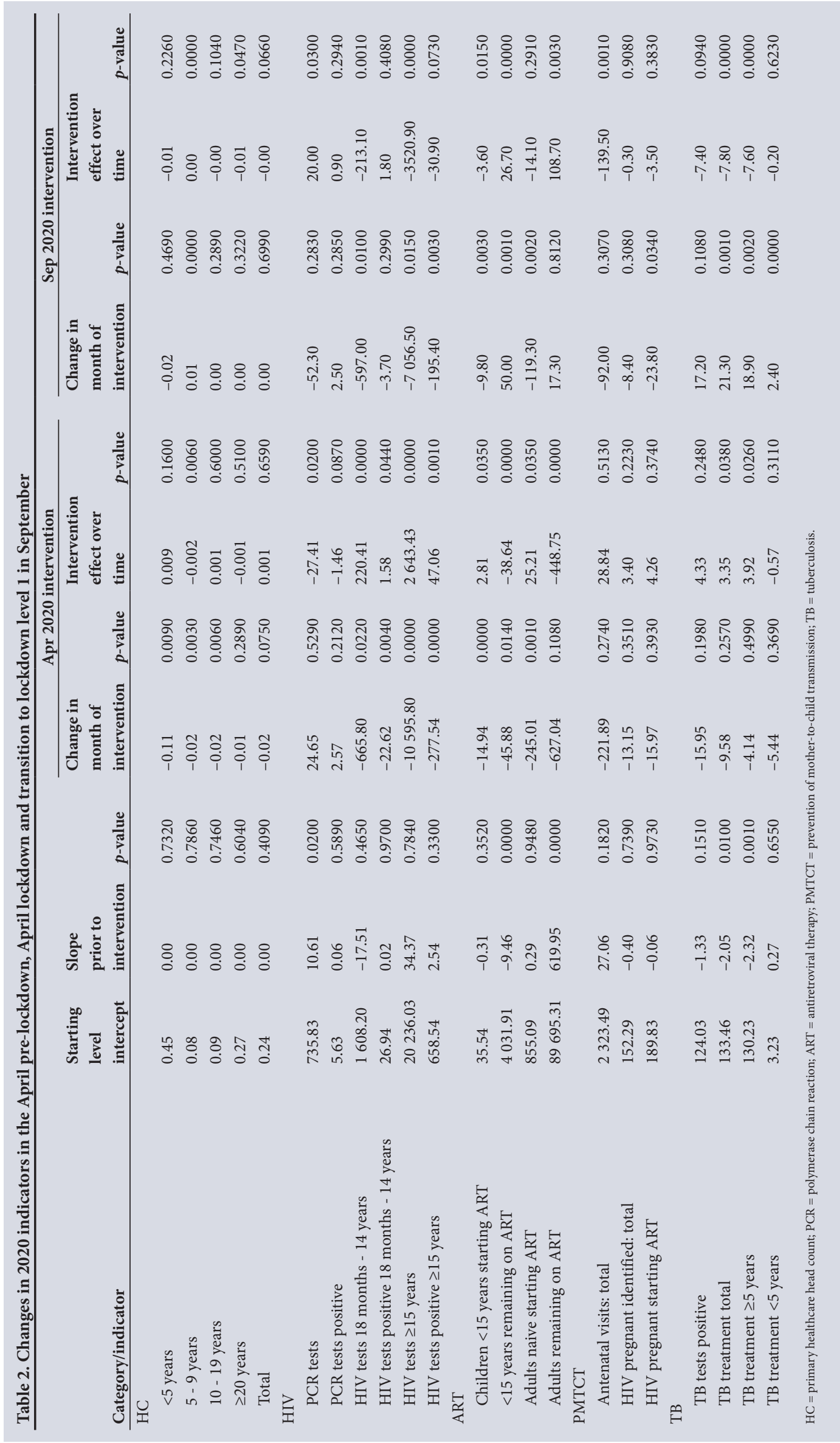




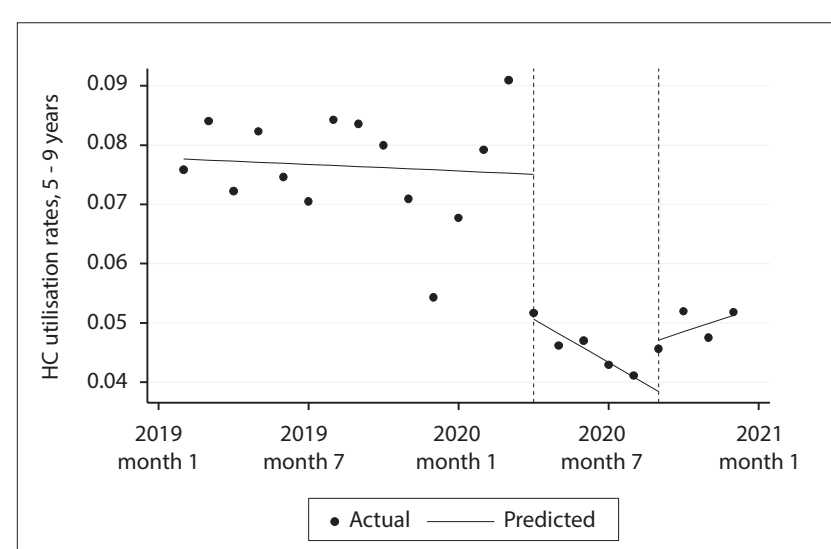

Regression with Newey-West standard errors - lag(3)

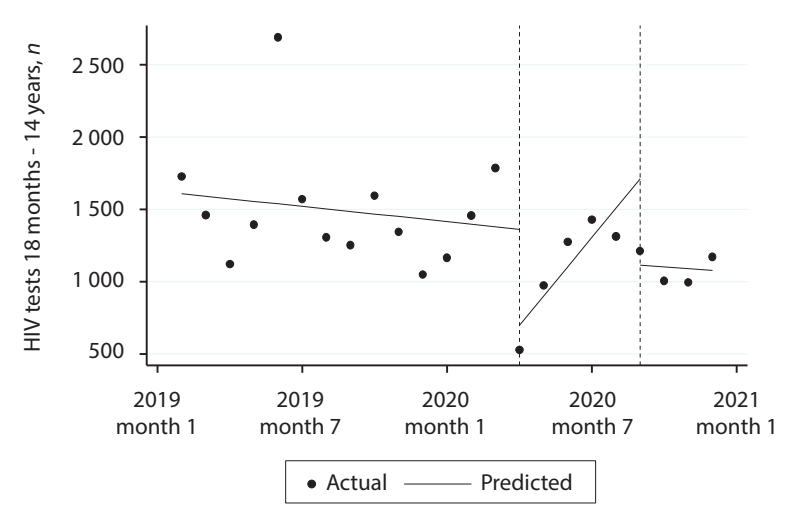

Regression with Newey-West standard errors - $\operatorname{lag}(2)$

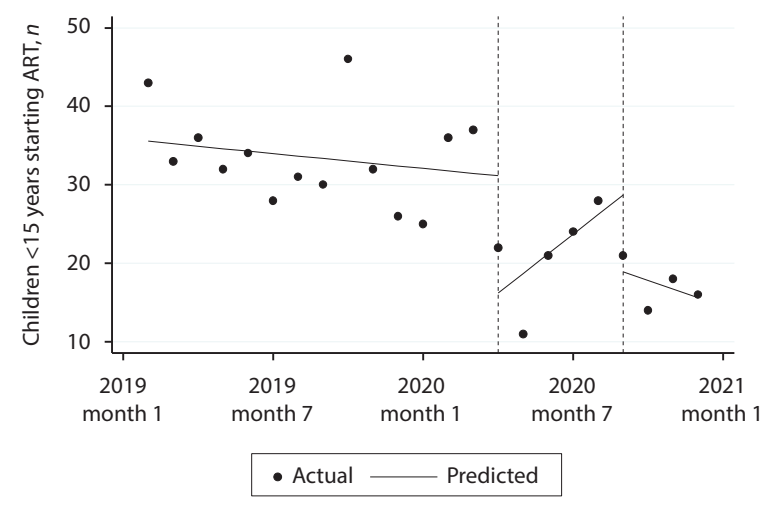

Regression with Newey-West standard errors - $\operatorname{lag}(3)$

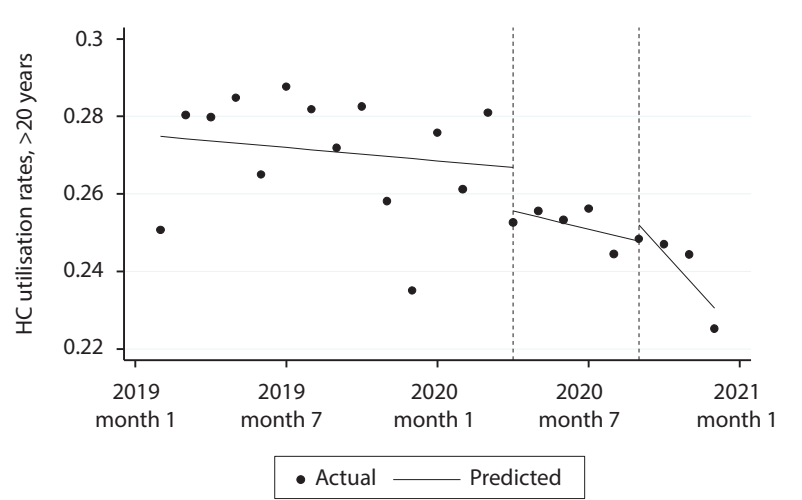

Regression with Newey-West standard errors - lag(1)

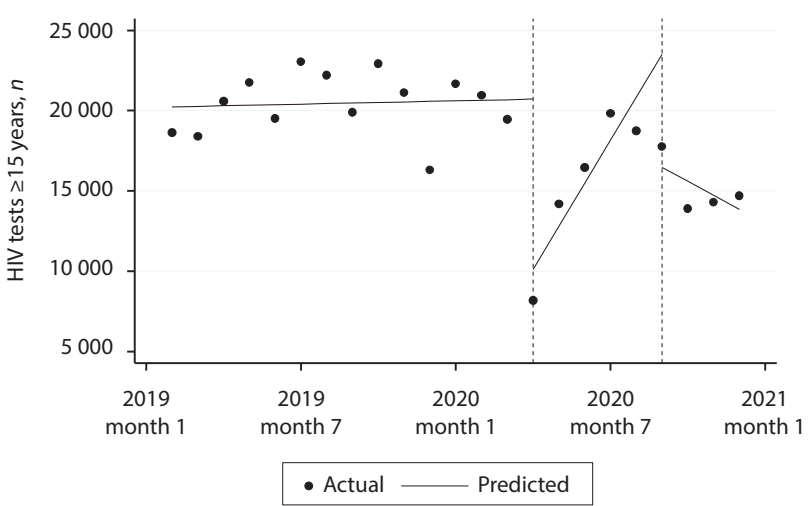

Regression with Newey-West standard errors - $\operatorname{lag}(1)$

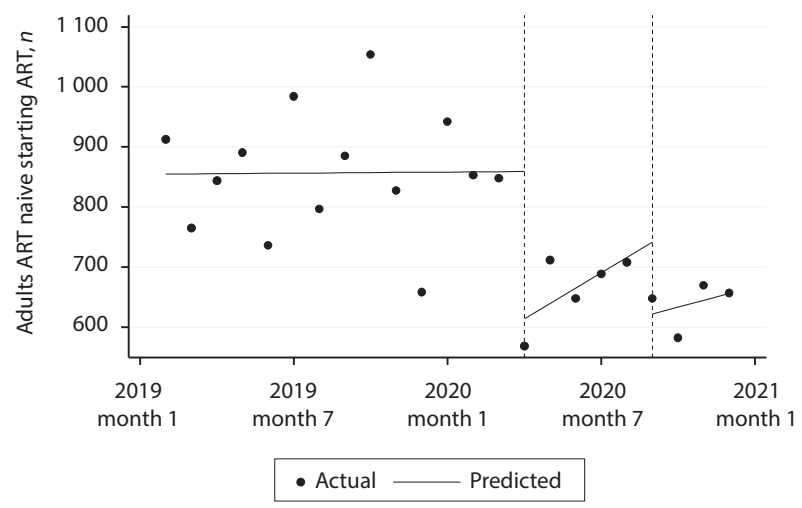

Regression with Newey-West standard errors - lag(1)

Fig. 1. Routine service delivery at the beginning of the April lockdown in 2020 and at the end of the first wave in September (indicated by the dashed vertical lines) for selected indicators. (HC = primary healthcare head count; $A R T=$ antiretroviral treatment; lag(1), (2) and (3) specify the number of lags considered in the autocorrelation structure.)

ART (1\%) indicators declined during the April lockdown, with no recovery during the change to level 1 until December 2020.

Table 2 shows that the lockdown in April 2020 contributed to statistically significant declines of 15 for children starting ART $(p=0.01), 46$ for children remaining on ART $(p=0.01)$ and 245 for adults starting ART indicators $(p=0.01$ ) (see also the trend lines in Fig. 1). The monthly trend after the lockdown shows statistically significant reductions for those remaining on ART indicators and statistically significant increases for those starting ART. In the month of the move to lockdown level 1, it appeared that ART enrolment indicators experienced a statistically significant decrease, while children $<15$ years remaining on ART experienced a statistically significant increase. The lockdown change was followed by statistically significant monthly increases for remaining on ART indicators and statistically significant monthly decreases for children $<15$ years starting ART. 


\section{PMTCT indicators}

There were no statistically significant changes for total antenatal visits and total pregnant HIV-positive women identified for the period April - December 2019 v. April - December 2020. Analysis of the quarterly data for January - March 2020 v. April - June 2020 showed modest decreases in the PMTCT indicators. Total antenatal visits appeared to show some improvement for the period May - October 2020, before decreasing in November.

The ITSA indicated no statistically significant effects of the lockdown in April 2020 (Table 2). However, at the end of the first wave, for HIV-positive pregnant women starting ART, we noted a statistically significant decline of 24 women $(p=0.05)$. The end of wave 1 was followed by statistically significant declines in the monthly trend in total antenatal visits $(p=0.05)$.

\section{TB indicators}

For the TB indicators, we included data on total positive TB cases and total clients enrolled on TB treatment. For those on TB treatment, we also present results disaggregated by age for children aged $<5$ years and those aged $\geq 5$ years. We found statistically significant declines in the total enrolled in TB treatment for April - December 2020 when compared with April - December 2019, with the largest decline being experienced in July 2020 (-26\%). Data show decreases across all TB indicators in April, the month of the first COVID-19 lockdown, with positive TB tests experiencing the largest decline $(-33 \%)$. Month-on-month analysis for TB tests positive and TB treatment total showed fluctuating trends, with another dip occurring in July 2020 after an improvement to almost pre-lockdown numbers in May. An improvement to almost pre-lockdown levels in September was notable. This was then followed by decreases in numbers.

While we did find decreases in all TB indicators during the April 2020 lockdown, results in Table 2 indicate no statistically significant lockdown effects. The lockdown was followed by a statistically significant increase in the monthly trend for total on TB treatment $(p=0.05)$. There is evidence of an increase in total on TB treatment in September, the month of the move to alert level $1(p=0.05)$. For both TB tests positive and TB treatment total, we then observed an additional decrease in monthly trends after the move to lockdown level 1.

\section{Discussion}

Previous studies show that the COVID-19 pandemic has significantly affected global economies and disrupted healthcare systems across the world. We present findings from an analysis of DHIS data and show varying levels of lockdown effects on HIV, TB and PMTCT indicators in Mopani district.

In contrast to findings from an ITSA in 11 primary care clinics in rural KwaZulu-Natal Province that found no change in the overall number of visits at the start of the lockdown, ${ }^{[13]}$ we observed a large statistically significant decline in health service utilisation when assessed using the total HC indicators. Our finding is consistent with a study that also used the DHIS data for the whole of SA and found a significant decline in primary healthcare in 2020 when compared with 2019 for all provinces. ${ }^{[2]}$ We observed no recovery after this lockdown or during the change to alert level 1 in September, and a further worsening in December. These trends did not differ markedly when disaggregated by age, except for HC for the age group 5 - 9 years, which although it experienced a large decline at the onset of COVID-19, appeared to improve slightly during the second wave in December 2020. Children aged 5 - 9 years do not have routine child health clinic visits (as do those aged $<5$ years), and they are not likely to have chronic diseases or sexual and reproductive health needs. The decline in HC in this age group was therefore probably due to a reduction in health-seeking for acute illnesses and injuries of mild to moderate severity. Our findings for the rest of the $\mathrm{HC}$ indicators suggest that clinic visits have not returned to pre-lockdown levels, demonstrating the huge lockdown effects. Other studies suggest that the decreases in $\mathrm{HC}$ are a result of movement restrictions, fear, stigma and misinformation, which prevent patients from coming to healthcare facilities. ${ }^{[24-26]}$ Findings from NIDS-CRAM (National Income Dynamics Study - Coronavirus Rapid Mobile Survey) show that COVID-19 and lockdown-related fears were the primary reason respondents with acute healthcare needs did not seek care. ${ }^{[9]}$

Subcategory analysis of HIV and ART indicators showed varying levels of effect. PCR tests and PCR tests positive appeared relatively unaffected by the pandemic lockdown levels. This is contrary to findings from a study by Jensen and McKerrow, ${ }^{[11]}$ which found that the infant PCR at birth uptake rate showed small but statistically significant declines. We suspect that the PCR indicator was unaffected because it forms part of the PMTCT programme, which we saw was not affected by the lockdown. HIV testing, on the other hand, was affected by the lockdown, as it is often provided to people visiting health facilities for other reasons. HIV testing for the 18 months - 14 years and $\geq 15$ years age groups appeared to follow similar trends. Both were significantly affected by the April 2020 lockdown and showed an improvement in monthly trend after April, before declining further at the end of the first wave and showing no improvement after September. Our data show that testing had still not returned to pre-COVID levels by December. The immediate negative effect of the lockdown is also reflected in other studies. ${ }^{[9,12]}$ Dorward et al. ${ }^{[12]}$ also found a decline (48\%) in HIV testing at the time of the national lockdown and a gradual improvement in testing volumes to pre-lockdown levels by July. National data reported in a study by Pillay et al..$^{[2]}$ also show that all provinces except North West experienced declines in the number of HIV tests conducted from March to December 2020 compared with the same period in 2019. Decreased HIV testing in health facilities in Mopani is a concern, as ensuring that people living with HIV have been tested and started on ART prevents HIV transmission, illness and death.

Our study showed statistically significant declines in ART initiation, both at the beginning of the first wave in April and at the move to lockdown level 1 in September. This finding is contrary to the finding in a study by Siedner et al. ${ }^{[13]}$ of a $20 \%$ increase in HIV-related healthcare (including ART initiations, ART collection and chronic care medical visits) immediately after the nationwide lockdown in April 2020. The authors suspected that this immediate jump was a result of a rush to collect medications before an anticipated drug shortage or limited access to healthcare. Other studies from Uganda and SA found that COVID-19 negatively affected chronic treatment adherence. ${ }^{[27,28]}$ The SA study, conducted by the Human Sciences Research Council, found that during the lockdown, 13\% of respondents did not have access to their chronic medication. ${ }^{[27]}$ Consistent with findings from Uganda, the COVID-19 pandemic and lockdown measures put in place impeded access to clinical care or community-based support, thus disrupting treatment adherence. ${ }^{[28,29]}$ Medication non-adherence has the potential of leading to more HIV-related deaths and reversing a country's previous gains in the diagnosis and treatment of HIV. Modelling studies indicate that a 6-month disruption in ART would result in an additional 500000 HIV-related adult deaths over 4 years in sub-Saharan Africa. ${ }^{[17]}$

We found no evidence of a statistically significant effect of the lockdown on PMTCT and TB indicators. The emphasis on a successful 
PMTCT programme may have contributed to the maintenance of service utilisation in Mopani. Antenatal visits remained relatively steady during the study period, although there was a marked increase in May 2020. This increase may have been due to pregnant women returning to their homes in rural areas at the end of alert level 5 lockdown. ${ }^{[30]}$ To date, few studies have analysed the impact of COVID-19 and the associated lockdown measures on PMTCT or TB indicators in SA. A study by Pattinson et al. ${ }^{[30]}$ found a 30\% increase in maternal deaths in SA during the country's first COVID-19 wave, compared with a similar period in 2019. The authors suspect that lack of transport, human resources and beds at facilities were among the leading causes of death. ${ }^{[30]}$ Siedner et al. ${ }^{[13]}$ found no evidence of drops in visits for perinatal care. An SA pre-exposure prophylaxis (PrEP) cohort study of pregnant and postpartum women in care found that during the nationwide lockdown, missed PrEP visits in the first month increased by $34 \%{ }^{[31]}$ Lockdown measures instituted to flatten the curve would be a massive setback in the delivery of PMTCT health services; however, we did not find any lockdown effects on PMTCT health services in our study. Modelling studies indicate that a 6-month disruption in ART would result in mother-to-child transmission of HIV doubling over 4 years in sub-Saharan Africa. ${ }^{[17]}$

The decreases in TB indicators during the April 2020 lockdown are consistent with National Health Laboratory Service data showing a $50 \%$ decline in $\mathrm{TB}$ tests during the level 5 lockdown in April 2020 , which was matched by a $33 \%$ decline in weekly averages for confirmed TB cases. ${ }^{[32]}$ The first national TB survey in SA found that many people with TB delay or do not seek treatment. ${ }^{[33]}$ Treatment interruptions and delays are what drive the TB epidemic in SA and are likely to affect any previous improvements in reducing $\mathrm{TB}$ incidence. Modelling studies indicate that a $25 \%$ reduction in TB detection for 3 months would result in a 13\% increase in TB-related deaths, resulting in an additional 1.4 million TB deaths between 2020 and 2025 due to the COVID-19 pandemic. ${ }^{[34]}$

\section{Study strengths and limitations}

A key strength of our study is the use of a large, standardised dataset that includes all public health facilities in Mopani district. This has the advantage of reflecting a true picture of the impact of COVID-19 on HIV, TB and PMTCT indicators in Mopani. Our study also has some limitations. Firstly, we focused on the effect of COVID-19 in Mopani, so our results may not be generalisable to other rural areas that experienced different COVID-19 infection rates. Secondly, the DHIS data collection and capturing is subject to human errors in data capturing because of the manual capturing process. These may have been exacerbated during the COVID-19 pandemic, with sickness and high workplace stress experienced. Data used in the analysis were captured during a period when resources were diverted to treating COVID-19 patients and may therefore have been subject to other inaccuracies such as missing or invalid data. Nevertheless, we attempted to mitigate the risk of data inaccuracy via querying outliers.

\section{Conclusions}

While healthcare systems have been affected by the pandemic, there is a need to achieve a balance between the fight against COVID19 and maintaining routine essential primary health services. The present study, using DHIS data, shows that the impact of the COVID-19 lockdown measures on health indicators varied. We observed that while HC, HIV and ART indicators were affected by the lockdowns, PMTCT and TB indicators were mostly unaffected. After an initial drop in $\mathrm{HC}$ rates, we observed no recovery in clinic visits well into the country's second wave in December 2020. The disruptions to HIV and ART indicators in our study are a cause for concern. The timely evidence provided by this study is important for strategies to be put in place to mitigate the effects of future COVID19 waves and lockdowns and encourage health-seeking behaviour. Our study highlights the importance of resilient healthcare systems and continued access to care, even in the context of a public health emergency. There is an urgent need for the investigation and use of alternative innovative service delivery options such as differentiated service delivery options, to ensure that future pandemics do not interrupt service delivery to the same extent. There is also a need to extend this research to establish the effect of the second wave and subsequent waves on HIV-, TB- and PMTCT-related health services.

\section{Declaration. None.}

Acknowledgements. None.

Author contributions. CM: data analysis and manuscript drafting. JD: review of the data analysis and critical review of the manuscript, CM, LR, TC, RN, HS and JAM: critical review of analysis and manuscript. KR: review of study design, data analysis and critical review of the manuscript. All authors provided approval for the version to be published.

Funding. This study was made possible by the support of the US President's Emergency Plan for AIDS Relief (PEPFAR) through the United States Agency for International Development (USAID) under Cooperative Agreement number 674-A-12-00015 to the Anova Health Institute. Anova Health Institute is a USAID-funded PEPFAR district support partner for the Mopani district in Limpopo Province, SA. As such, Anova provides technical assistance at public sector facility level and directs service delivery via healthcare workers employed by Anova. The contents are the responsibility of Anova Health Institute and do not necessarily reflect the views of USAID or the US Government.

Conflicts of interest. None.

1. Banerjee I, Robinson J, Sathian B, van Teijlingen ER. South Africa and its COVID-19 prohibition predilection. Nepal J Epidemiol 2020;10(3):874-877. https://doi.org/10.3126/nje.v10i3.31543

Pillay Y, Pienaar S, Barron P, Zondi T. Impact of COVID-19 on routine primary healthcare services in South Africa. S Afr Med J 2021;111(8):714-719. https://doi.org/10.7196/SAMJ.2021.v111i8.15786 3. World Health Organization. WHO coronavirus disease (COVID-19) dashboard. 2021. https://covid19. 3. World Health Organization. WHO coronavirus
who.int/explorer (accessed 25 February 2021).

4. Martin DP, Weaver S, Tegally H, et al. The emergence and ongoing convergent evolution of the N501Y lineages coincides with a major global shift in the SARS-CoV-2 selective landscape. medRxiv 2021 (epub 25 July 2021). https://doi.org/10.1101/2021.02.23.21252268

5. Haider N, Osman AY, Gadzekpo A, et al. Lockdown measures in response to COVID-19 in nine sub-Saharan African countries. BMJ Glob Health 2020;5(10):e003319. https://doi.org/10.1136/ bmjgh-2020-003319

6. Broadbent A, Combrink H, Smart B. COVID-19 in South Africa. Glob Epidemiol 2020;2:100034. https://doi.org/10.1016/j.gloepi.2020.100034

7. Republic of South Africa. President Cyril Ramaphosa: South Africa's response to Coronavirus Covid-19 pandemic. 23 April 2020. https://www.gov.za/speeches/president-cyril-ramaphosa-southafricas-response-coronavirus-covid-19-pandemic-23-apr-2020 (accessed 9 April 2021).

8. Mayosi BM, Flisher AJ, Lalloo UG, Sitas F, Tollman SM, Bradshaw D. The burden of nonMayosi BM, Flisher AJ, Lalloo UG, Sitas F, Tollman SM, Bradshaw D. The burden of non-
communicable diseases in South Africa. Lancet 2009;374(9693):934-947. https://doi.org/10.1016/ communicable diseases

9. Burger R, Day C, Deghaye N, et al. Examining the unintended consequences of the COVID-19 pandemic on public sector health facility visits: The first 150 days. Wave 2 Report. National Income Dynamics Study (NIDS) - Coronavirus Rapid Mobile Survey (CRAM). 2020. https://cramsurvey.org/wp-content/ uploads/2020/12/16.-Examining-the-unintended-consequences-of-the-COVID-19-pandemic-onpublic-sector-health-facility-visits-The-first-150-days-2.pdf (accessed 22 February 2021).

10. Hofman K, Madhi S. The unanticipated costs of COVID-19 to South Africa's quadruple disease burden. S Afr Med J 2020;110(8):689-699. https://doi.org/10.7196/SAMJ.2020.v110i8.15125

11. Jensen C, McKerrow N. Child health services during a COVID-19 outbreak in KwaZulu-Natal Province, South Africa. S Afr Med J 2020;111(2):114-119. https://doi.org/10.7196/SAMJ.2021. v111i2.15243

12. Dorward J, Khubone T, Gate K, et al. The impact of the COVID-19 lockdown on HIV care in 65 South African primary care clinics: An interrupted time series analysis. Lancet HIV 2021;8(3):E158-E165. http:///oi.org/10.1016/S2352-3018(20)30359-3

13. Siedner MJ, Kraemer JD, Meyer MJ, et al. Access to primary healthcare during lockdown measures 3. Siedner MJ, Kraemer JD, Meyer MJ, et al. Access to primary healthcare during lockdown measures
for COVID-19 in rural South Africa: A longitudinal cohort study. medRxiv 2020 (epub 20 May 2020). for COVID-19 in rural South Africa: A longit

14. National Institute for Communicable Diseases. Impact of COVID-19 intervention on TB testing in South Africa. 10 May 2020. https://www.nicd.ac.za/wp-content/uploads/2020/05/Impact-of-Covid19-interventions-on-TB-testing-in-South-Africa-10-May-2020.pdf (accessed 26 February 2021). 
15. Statistics South Africa. Mortality and causes of death in South Africa: Findings from death notification. Statistical release P0309.3. Pretoria: Stats SA, 2017. https://www.statssa.gov.za/publications/P03093/ P030932017.pdf (accessed 25 February 2021)

16. World Health Organization. South Africa - HIV country profile: 2018. Geneva: WHO, 2019. http://cfs. hivci.org/country-factsheet.html (accessed 26 February 2021).

17. Jewell BL, Mudimu E, Stover J, et al. Potential effects of disruption to HIV programmes in subSaharan Africa caused by COVID-19: Results from multiple mathematical models. Lancet HIV 2020;7(9):E629-E640. https://doi.org/10.1016/\$2352-3018(20)30211-3

18. Mopani District Municipality. Reviewed Integrated Development Plan 2016 - 2021 (2020/21 version 5). 2020. http://www.mopani.gov.za/docs/idp/Mopanii\%20DM\%202020\%202021\%20Version\%205 pdf (accessed 14 April 2021)

19. IBM Research South Africa. COVID-19 data visualistion tool. https://gpcoronavirus.mybluemix net/2020 (accessed 22 February 2021)

20. Bradshaw D, Laubscher R, Dorrington R, Groenewald P, Moultrie T. Report on weekly deaths in South Africa: 25 April - 1 May 2021 (week 17). Cape Town: Burden of Disease Research Unit, South African Medical Research Council 4 May 2021. https.//www.samrc.ac.za/sites/default/files/ files/2021-05-05/weekly1May2021.pdf (accessed 10 May 2021).

21. Linden A. Conducting interrupted time-series analysis for single-and multiple-group comparisons. Stata J 2015;15(2):480-500. https://doi.org/10.1177/1536867X1501500208

22. Baum C, Schaffer M. IVACTEST: Stata module to perform Cumby-Huizinga test for autocorrelation after IV/OLS estimation. Statistical software components. Revised 24 January 2015. https://econpapers repec.org/software/bocbocode/s457668.htm (accessed 15 February 2021).

23. Statistics South Africa. Provincial profile: Limpopo. Community Survey 2016. Report 03-01-15. Pretoria: Stats SA, 2018. http://cs2016.statssa.gov.za/wp-content/uploads/2018/07/Limpopo.pdf (accessed 2 December 2020).

24. Ponticiello M, Mwanga-Amumpaire J, Tushemereirwe P, et al. 'Everything is a mess': How COVID-19 is impacting engagement with HIV testing services in rural southwestern Uganda. AIDS Behav 2020;24:3006-3009. https://doi.org/10.1007/s10461-020-02935-w

25. Lagat H, Sharma M, Kariithi E, et al. Impact of the COVID-19 pandemic on HIV testing and assisted partner notification services, Western Kenya. AIDS Behav 2020;24:3010-3013. https://doi.org/10.1007/ s10461-020-02938-7
26. Nyasulu J, Pandya H. The effects of coronavirus disease 2019 pandemic on the South African health system: A call to maintain essential health services. Afr J Prim Health Care Fam Med 2020;12(1):a2480. hystem: A call to maintain essential health

27. Human Sciences Research Council. HSRC study on COVID-19 indicates overwhelming compliance with the lockdown. 26 April 2020. http://www.hsrc.ac.za/en/media-briefs/general/lockdown-surveyresults (accessed 8 December 2020).

28. Linnemayr S, Mayo-Wilson LJ, Saya U, et al. HIV care experiences during the COVID-19 pandemic: Mixed-methods telephone interviews with clinic-enrolled HIV-infected adults in Uganda. AID Behav 2021;25:28-39. https://doi.org/10.1007/s10461-020-03032-8

29. Nyoni T, Okumu M. COVID-19-compliant strategies for supporting treatment adherence among people living with HIV in sub-Saharan Africa. AIDS Behav 2020;24:2473-2476. https://doi. org/10.1007/s10461-020-02888-0

30. Pattinson R, Soma-Pillay P, Moodley J, Fawcus S, Gebhardt S, Niit R. The effect of the first wave of Covid-19 on use of maternal and reproductive health services and maternal deaths in South Africa. Obstetrics and Gynaecology Forum 2020;30(4):38-46.

31. Davey DLJ, Bekker L-G, Mashele N, Gorbach P, Coates TJ, Myer L. PrEP retention and prescription for pregnant women during COVID-19 lockdown in South Africa. Lancet HIV 2020;7(11):E735. for pregnant women during COV18-19 lock-5

32. Madhi S, Gray G, Ismail N, et al. COVID-19 lockdowns in low-and middle-income countries: Success against COVID-19 at the price of greater costs. S Afr Med J 2020;110(8):724-726. https://doi org/10.7196/SAMJ.2020.v110i8.15055

33. National Department of Health, South Africa. The First National TB Prevalence Survey: South Africa 2018. Short report. https://www.knowledgehub.org.za/system/files/elibdownloads/2021-02/A4_SA TPS\%20Short\%20Report_10June20_Final_highres.pdf (accessed 12 April 2021).

34. Glaziou P. Predicted impact of the COVID-19 pandemic on global tuberculosis deaths in 2020 medRxiv 2021 (epub 8 October 2021). https://doi.org/10.1101/2020.04.28.2007958

Accepted 17 August 2021 